\title{
A concise review on multi-agent teams: contributions and research opportunities ${ }^{\star}$
}

\author{
Ewa Andrejczuk ${ }^{1,2}$, Juan A. Rodriguez-Aguilar ${ }^{1}$, Carles Sierra ${ }^{1}$ \\ 1 Artificial Intelligence Research Institute (IIIA-CSIC), Barcelona, Spain \\ ${ }^{2}$ Change Management Tool S.L., Barcelona, Spain \\ \{ewa,jar,sierra\}@iiia.csic.es
}

\begin{abstract}
The composition and formation of effective teams is crucial for both companies, to assure their competitiveness, and for a broad range of emerging applications exploiting multiagent collaboration (e.g. human-agent teamwork, crowdsourcing). The aim of this article is to provide an integrative perspective on team composition, team formation and their relationship with team performance. Thus, we review and classify the contributions in the computer science literature dealing with these topics. Our purpose is twofold. First, we intend to identify the strengths and weaknesses of the contributions made so far. Second, we pursue to identify research gaps and opportunities. Given the volume of the existing literature, our review is not intended to be exhaustive. Instead, we focus on the most recent contributions that broke new ground to spur innovative research.
\end{abstract}

\section{Introduction}

In the last decades, there has been increasing interest in team-based work structures together with a focus on organisational efficiency [16]. On that account, team composition and formation research is of interest to many fields of science, also of computer science, especially within the area of multiagent systems (MAS). In this paper, our understanding of team composition and formation differentiates from the definitions provided by the multiagent field. We define team composition as the process of deciding which agents will be part of a team. We understand team formation as the process undertaken by agents to learn to work together in a team, and through this learning decide the roles and internal organisation of the team. Our definition of team formation is in line with the organisational psychology literature, which differentiates between the team composition and formation processes [16, p.16].

The aim of this article is: (i) to determine dimensions that will help to classify MAS literature; (ii) to survey the most recent contributions in the literature on team composition and formation according to identified dimensions; and (iii) to identify research gaps and opportunities by classifying the current state-of-the-art on team composition and formation.

* Work supported by Collectiveware TIN2015-66863-C2-1-R (MINECO/FEDER), CollectiveMind (MINECO TEC2013-49430-EXP), SMA (201550E040), and Gencat 2014 SGR 118. Ewa Andrejczuk is supported by an Industrial PhD scholarship from the Generalitat de Catalunya. 
In order to structure our analysis, we have identified several dimensions that will help us dissect the contributions in the literature:

1. WHO is concerned? The properties of the agents involved.

2. WHAT is the problem? The features of the task to complete by a team.

3. WHY do we do it? The objective function to optimise when composing/forming a team.

4. HOW do we do it? The organisation and/or coordination structure adopted by the team in charge of performing a particular task.

5. WHEN do we do it? The dynamics of the stream of tasks to be completed by agent teams.

Overall, our analysis of the literature indicates that MAS research focuses on building systems whose agents interact to achieve a common objective or exploit each other features to achieve self-interested goals. The concept of agent teams is quite simplistic and it does not include the whole complexity of aspects considered by the organizational psychology (OP) literature. For instance, OP assumes that human capabilities are necessarily dynamic [17] (evolve along time) so that teams can successfully perform tasks in dynamic real-world scenarios and in a variety of contexts. Furthermore, OP observes that the quality of human resources (e.g. motivation, satisfaction, commitment), the ability of individuals to learn new capabilities, and the context constraining a team significantly influence its performance $[25,26,11,12]$. The MAS literature has typically disregarded significant organizational psychology findings, with the exception of several recent, preliminary attempts (such as [10], [13], [3]). However, our analysis of the literature indicates that Computer Science (CS) and OP exhibit also some similarities. One of the crucial findings in CS that has been confirmed by organizational psychology studies [32] is that team members have to be heterogeneous to maximize team performance. When modeling agents, similarly to OP, the Computer Science (CS) literature considers two main approaches: either there is complete information about the properties of each agent; or agents are capable of learning about their teammates through repeated interactions. We believe that further analysis of the OP literature could be beneficial to CS.

For a long version of this article, containing both a review of the OP literature review and a detailed description for each dimension above, we refer the reader to [4].

\section{Dimensions to analyse the state of the art}

In this section we describe the meaning of each of the dimensions identified in the introduction above.

\subsection{WHO is concerned?}

MAS research mainly focuses on the interaction among intelligent agents. In the team composition and formation literature, the focus is on the interaction of cooperative and 
heterogeneous agents. That is, agents who share a common goal, but have different individual properties. Here we classify the literature depending on the agents' individual properties along two dimensions: capacity and personality.

2.1.1 Capacity: individual and social capabilities of agents. In many domains, a capability is defined as a particular skill required to perform an action. The capacity dimension has been exploited by numerous previous works $[2,6-8,18,23,24,28]$. The majority of these approaches represent capabilities of agents in a Boolean way (i.e., an agent either has a required skill or not). When modeling agents' properties, many existing approaches typically assume extensive a-priori information about teammates (e.g. [2, 8, 15, 28] just to name a few). This is a strong limitation for real-life settings. Notice that in many companies there is no central and extensive knowledge about all employees' capabilities.

2.1.2 Personality: Individual behaviour models. Very recently some MAS contributions have started to consider the notion of personality, i.e. individual behaviour model, to compose heterogeneous teams $[1,3,5,9,10,13,20-22]$. We observe an increasing interest in building more realistic models considering agents' behavioural patterns.

\subsection{WHAT is the problem? The notion of task}

In its most general sense, a task is a course of action to achieve a goal. The execution of a task is then usually equated to the execution of an action plan. In the team composition and formation literature it is often the case that simplifying assumptions are made and tasks are assumed to be solved by simple action plans. For instance, an action plan can be seen as a set of actions, or even as a set of competences. Thus, we identify two main approaches: individual-based and plan-based.

2.2.1 Individual-based approaches. Given a task, it is generally assumed that if the joint capabilities of agents in a team fulfill those required by the task, then the team is capable of solving the task. Existing work on team composition focuses on two categories of individual properties: capacity and personality. Regarding capacity, there are many models in the MAS literature that define a task as a set of requirements on agents' capacities. These requirements are either direct (a task makes explicit a set of demanded capabilities $[2,7,8,23]$ ), or indirect (sub-tasks are matched to agents' capacities [24] or task complexity levels are matched to graded agents' capabilities [6]). Regarding personality, some works define task types and match them with different personalities $[10,9]$. Others highlight the importance of diversity in personalities within a team [3] or communication style (associated with personality type) [13]. 
2.2.2 Plan-based approaches. The notion of task in plan-based approaches is normally understood either as a set of actions or as a sequence of actions that are assigned to the individual members of a team. Some authors [5, 1] employ an indirect planning method driven only by the most informed agents to solve a set of actions. Other approaches consider a task as a sequence of actions and let all agents in a team jointly vote on the possible alternatives from a discrete set of possible actions [20,22,21].

\subsection{WHY do we do it? The objective(s)}

The motivation of individual efforts or actions is to attain or accomplish a certain state of affairs: a goal. A large body of the literature proposes team composition and formation algorithms to attain at least one of the following team objectives: minimizing overall cost (e.g. cooperation cost, team cost), maximizing social utility, or maximizing the quality of an outcome. Regarding the first objective, there are various costs associated with team composition and formation problems (e.g. communication costs, or agent service costs). The reviewed models in the MAS literature minimizing overall cost $[8,15,23]$ compose teams based on individual competences, though do not take into account individual motivations to complete some assigned task. A second objective considered in the team composition and formation literature is maximizing social welfare. That is, maximizing the utility function of a team. Typically, the utility obtained is then allocated to the individual members of the team $[1,6,7,10,30]$. The literature focusing on maximizing social welfare considers both agent competences and motivation. Motivation increases by making agents compete (like in crowdsourcing teams [30]), or by giving agents the freedom to select their collaborators (like in [6] or [1]). Finally, researchers in MAS propose a number of methods where agents try to maximize the quality of solutions whilst minimizing the time to achieve them, namely to maximize team performance [3, 13, 18-20,24,29]. To do this, one of the crucial findings in CS is that team members must be heterogeneous. Further variables that have been used by computer scientists in the area of MAS to compose teams are: agent reputation [24], personality of humans and agents [3,10,20], synergy between team members [19], and feeling of fairness among team members [29].

\subsection{HOW do we do it? The organisation}

There are two aspects to be considered while discussing the societal structure of teams, that is: which agents will be members of a team, and how teams will be organized to solve tasks.

2.4.1 Team Composition is the process of deciding which agents will be part of a team. Although team composition in MAS has mainly focused on building teams of software agents, that is, agent teams, there is a growing number of works considering either mixed teams [13], where agents and humans cooperate to achieve common goals [27], or human environments, where people are supported by software [14]. In MAS, we distinguish 
between two groups of methods (or processes) to compose teams: exogenous and endogenous. Exogenous team composition is when there exists an algorithm external to the agents that determines the composition of teams. The majority of reviewed works focuses on these methods to compose either the best team for a given task $[8,23,19,28,7]$ or a set of teams to solve an incoming set of tasks $[2,3,9,31]$. Endogenous methods for organizing teams incorporate algorithms enabling agents to decide on team composition by themselves. In detail, agents are equipped with negotiation and decision-making mechanisms that they employ to agree among themselves on a team structure $[10,24,6]$. Therefore, team composition occurs without explicit external command and in a distributed manner.

2.4.2 Team Formation is the process of deciding the roles and internal organisation of a team. This organisation can be imposed or be the result of self-organisation. The resulting organisations can be categorized as hierarchical or egalitarian. A hierarchical structure considers a team leader who is responsible for and makes the decisions affecting the team. This organisation type is imposed by defining two or more types of agents (such as requesters and contributors [10], mediators and workers [24], or best-response agents and ad-hoc agents [1]). An egalitarian structure assumes that all workers in a team are equally informed and have the same rights. The leadership within a team is shared and existing team roles result from the team's task requirements. We find this team structure in Groupsourcing [30], Robust Teams [8,23], Ad-hoc teams [5-7], Mixed Teams [13], Learning Teams $[18,19]$ or Voting Teams [20-22].

\subsection{WHEN do we do it? The dynamics}

The literature on team composition and formation mostly considers that tasks are static in the sense that their requirements do not change during their execution. However, some works consider that there is a stream of tasks that dynamically appear to be completed. Thus, there could be multiple tasks to be solved concurrently and new tasks may arrive in an asynchronous, localized manner. The different works in the literature consider different issues in this dynamic process. For instance, the number of tasks to be serviced, task and team members localization, team size per task or time limitations. Hence, the literature can be classified depending on two main aspects: the succession of tasks and the concurrency of tasks.

The simplest case is represented by a one-shot task. There is neither succession nor concurrency, and hence the problem of team composition is normally reduced to finding the best team for the only task $[8,15,23,28]$. When tasks come in sequence without concurrency, then the problem can be reduced to finding the best team for each task while using the learned experiences in the composition of each new team $[2,19]$. If tasks come in succession and can be simultaneous, the need for dealing with multiple teams acting

at the same time becomes the key issue. Finally, the succession of possibly simultaneous tasks represents the most complex scenario $[6,7,10,24]$. Here memory becomes a crucial 
element as it lets agents learn from the past experiences and build their beliefs based on this knowledge.

\section{Discussion}

The aim of this article is to review the most recent, representative and relevant literature on team composition and formation and identify research gaps and opportunities for further research. In this final section, we focus on identifying research opportunities:

- Establish a connection with the OP literature. A goal of organizational psychology is to improve organizational performance by placing the right people in the right jobs, thus enhancing the fit between the individual and the organization. This includes the methods for building effective teams. Nevertheless, research on team composition and team formation in CS and OP has evolved separately. The MAS literature has typically disregarded significant OP findings, with the exception of several recent, preliminary attempts (like [3], [10] or [13]). This body of research has focused on algorithms that help automate team formation and composition. Heuristics for team composition and formation investigated by the OP literature have much potential for MAS research.

- Exploration of complex agents. The CS literature is in need of analysing more complex examples where humans are modeled as agents. While some of the human properties may not make sense in an agent context, some do. For instance, the dynamics of competences through learning and experience and the cultural values could be used to program more sophisticated agents, specially when interacting in mixed teams involving humans. Additionally, OP research highlights motivation as an important factor for team performance [12]. The majority of MAS literature on team composition and teamwork assumes that agents always behave according to their capabilities and knowledge. While in MAS research it is shown that motivation increases by introducing competition mechanisms (like in crowdsourcing teams [30]), or by giving agents freedom when selecting their collaborators (like in ad-hoc teams [1]), there are only early attempts to include agents' motivation as an important factor for team performance.

- Study of plan-based approaches. Regarding the tasks that are executed by agent teams, CS focuses on team members' properties required to perform a task rather than on a detailed planning of task execution. The majority of approaches assume that the joint capabilities of agents in a team are enough to solve a given task. There are some preliminary attempts to include planning, though they are very simplistic. The majority of methods do not consider time constraints, action dependencies, action failure, plan robustness, task dynamic changes and hence, the vast literature on planning has not yet been integrated into team formation methods.

- Exploration of complex approaches for task execution. Since in CS agents can be engineered depending on the needs (i.e. agents can be designed with different properties, such as personality or memory, depending on the whole system design), researchers can study different settings depending on the dynamics of task arrival (one task or 
many, one time or many). The CS literature uses complex scenarios to let agents build their beliefs based on past experiences and compose new teams according to these learned beliefs. However, while executing tasks, there are no contributions that explore successive or simultaneous settings. Hence, the state of the importance of agent learning when executing tasks.

- The study of team properties. Although individuals' properties have been extensively studied and considered, there is still a need for modeling the global properties of agent teams. Such modeling should go beyond considering simple properties such as the sum of the agents' individual capabilities or the Boolean representation of whether the team can perform a task or not.

\section{References}

1. N. Agmon, S. Barrett, and P. Stone. Modeling uncertainty in leading ad hoc teams. In Proceedings of the 13th International Conference on Autonomous Agents and Multiagent Systems (AAMAS), May 2014.

2. A. Anagnostopoulos, L. Becchetti, C. Castillo, A. Gionis, and S. Leonardi. Online team formation in social networks. In Proceedings of the 21st World Wide Web Conference 2012, WWW 2012, Lyon, France, April 16-20, 2012, pages 839-848, 2012.

3. E. Andrejczuk, J.A. Rodríguez-Aguilar, and Sierra C. Optimising congenial teams. 10/05/2016 2016.

4. Ewa Andrejczuk, Rita Berger, Juan A Rodriguez-Aguilar, Carles Sierra, and Víctor Marín-Puchades. The composition and formation of effective teams. computer science meets psychology. arXiv preprint arXiv:1610.08804, 2016.

5. S. Barrett, P. Stone, S. Kraus, and A. Rosenfeld. Teamwork with limited knowledge of teammates. In Proceedings of the Twenty-Seventh AAAI Conference on Artificial Intelligence, July 2013.

6. G. Chalkiadakis and C Boutilier. Sequentially optimal repeated coalition formation under uncertainty. Autonomous Agents and Multi-Agent Systems, 24(3):441-484, 2012.

7. B. Chen, X. Chen, A. Timsina, and L. Soh. Considering agent and task openness in ad hoc team formation. In Proceedings of the 2015 International Conference on Autonomous Agents and Multiagent Systems, AAMAS 2015, Istanbul, Turkey, May 4-8, 2015, pages 1861-1862, 2015.

8. C. Crawford, Z. Rahaman, and Sen S. Evaluating the efficiency of robust team formation algorithms. International Workshop on Optimisation in Multi-Agent Systems, 2016.

9. M. Farhangian, M.K. Purvis, M. Purvis, and B.T.R. Savarimuthu. Agent-based modeling of resource allocation in software projects based on personality and skill. In Communications in Computer and Information Science, pages 130-146, 2015.

10. M. Farhangian, M.K. Purvis, M. Purvis, and B.T.R. Savarimuthu. Modeling the effects of personality on team formation in self-assembly teams. In PRIMA 2015: Principles and Practice of Multi-Agent Systems - 18th International Conference, Bertinoro, Italy, October 26-30, 2015, Proceedings, pages 538-546, 2015.

11. R.A. Guzzo and M.W. Dickson. Teams in organizations: Recent research on performance and effectiveness. Annual review of psychology, 47(1):307-338, 1996.

12. J.R. Hackman. Groups that work (and those that don't): Creating conditions for effective teamwork. Number 10-H123. Jossey-Bass, 1990.

13. N. Hanna and D. Richards. Do birds of a feather work better together? the impact of virtual agent personality on a shared mental model with humans during collaboration. In Proceedings of the 3rd International Workshop on Collaborative Online Organizations, COOS 2016, co-located with the 14th International Conference on Autonomous Agents and Multi-Agent Systems, AAMAS 2015, Istanbul, Turkey, May 4, 2015., pages 28-37, 2015. 
14. N.R. Jennings, L. Moreau, D. Nicholson, S. Ramchurn, S. Roberts, T. Rodden, and A. Rogers. Humanagent collectives. Communications of the ACM, 57(12):80-88, 2014.

15. M. Kargar, A. An, and M. Zihayat. Efficient bi-objective team formation in social networks. In Proceedings of the 2012 European Conference on Machine Learning and Knowledge Discovery in Databases - Volume Part II, pages 483-498, Berlin, Heidelberg, 2012. Springer-Verlag.

16. S.W.J. Kozlowski and B.S Bell. Work groups and teams in organizations: Review update. 2013.

17. Marjan Laal and Peyman Salamati. Lifelong learning; why do we need it? Procedia-Social and Behavioral Sciences, 31:399-403, 2012.

18. S. Liemhetcharat and M. Veloso. Team formation with learning agents that improve coordination. In Proceedings of the 2014 International Conference on Autonomous Agents and Multi-agent Systems, AAMAS '14, pages 1531-1532, Richland, SC, 2014. International Foundation for Autonomous Agents and Multiagent Systems.

19. S. Liemhetcharat and M.M. Veloso. Modeling and learning synergy for team formation with heterogeneous agents. In International Conference on Autonomous Agents and Multiagent Systems, AAMAS 2012, Valencia, Spain, June 4-8, 2012 (3 Volumes), pages 365-374, 2012.

20. L.S. Marcolino, A.X. Jiang, and A Tambe. Multi-agent team formation: Diversity beats strength? In IJCAI 2013, Proceedings of the 23rd International Joint Conference on Artificial Intelligence, Beijing, China, August 3-9, 2013, 2013.

21. L.S. Marcolino, H. Xu, D. Gerber, B. Kolev, S. Price, E. Pantazis, and M. Tambe. Multi-agent team formation for design problems. In Coordination, Organizations, Institutions and Norms in Agent Systems XI. Springer-Verlag Lecture Notes in AI, 2016.

22. V. Nagarajan, L.S. Marcolino, and M. Tambe. Every team deserves a second chance: Identifying when things go wrong (student abstract version). In Proceedings of the Twenty-Ninth AAAI Conference on Artificial Intelligence, January 25-30, 2015, Austin, Texas, USA., pages 4184-4185, 2015.

23. T. Okimoto, N. Schwind, M. Clement, T. Ribeiro, K. Inoue, and P. Marquis. How to form a taskoriented robust team. In Proceedings of the 2015 International Conference on Autonomous Agents and Multiagent Systems, AAMAS '15, pages 395-403. International Foundation for Autonomous Agents and Multiagent Systems, 2015.

24. A. Peleteiro, J.C. Burguillo-Rial, M. Luck, J.L. Arcos, and J.A. Rodríguez-Aguilar. Using reputation and adaptive coalitions to support collaboration in competitive environments. Engineering applications of artificial intelligence, 45:325-338, 2015.

25. Philip M Podsakoff, Scott B MacKenzie, and Michael Ahearne. Moderating effects of goal acceptance on the relationship between group cohesiveness and productivity. Journal of Applied Psychology, 82(6):974, 1997.

26. S Quijano, J Navarro, M Yepes, R Berger, and M Romeo. Human system audit (hsa) for the analysis of human behaviour in organizations. Papeles del Psicólogo, 29(1):92-106, 2008.

27. S.D. Ramchurn, F. Wu, J.E. Fischer, S. Reece, J. W, S.J. Roberts, T. Rodden, and N.R. Jennings. Human-agent collaboration for disaster response. Journal of Autonomous Agents and Multi-Agent Systems, pages 1-30, 2016.

28. S.S. Rangapuram, T. Bühler, and M. Hein. Towards realistic team formation in social networks based on densest subgraphs. CoRR, abs/1505.06661, 2015.

29. I. Rochlin, Y. Aumann, D. Sarne, and L. Golosman. Efficiency and fairness in team search with self-interested agents. Autonomous Agents and Multi-Agent Systems, 30(3):526-552, 2016.

30. M. Rokicki, S. Zerr, and S. Siersdorfer. Groupsourcing: Team competition designs for crowdsourcing. In Proceedings of the 24th International Conference on World Wide Web, WWW 2015, Florence, Italy, May 18-22, 2015, pages 906-915, 2015.

31. M. Spradling, J. Goldsmith, X. Liu, C. Dadi, and Z. Li. Roles and teams hedonic game. In ADT, volume 8176 of Lecture Notes in Computer Science, pages 351-362. Springer, 2013.

32. D.J. Wilde. Teamology: The Construction and Organization of Effective Teams. Springer-Verlag, London, 2009. 